The Free Internet Journal

for Organic Chemistry
Paper

Arkivoc 2017, part iv, 343-352

\title{
Appel reagent as novel promoter for the synthesis of polysubstituted imidazoles
}

\author{
Mehdi Khoshneviszadeh ${ }^{\mathrm{a}}$ and Mohammad Mahdavi ${ }^{\mathrm{b} *}$
}

${ }^{a}$ Medicinal\&Natural Products Chemistry Research Center, Shiraz University of Medicinal Sciences, Shiraz, Iran

${ }^{b}$ Endocrinology and Metabolism Research Center, Endocrinology and Metabolism Clinical Sciences Institute, Tehran University of Medical Sciences, Tehran

Email: mahdavi chem@yahoo.com

Received 01-30-2017

Accepted 05-06-2017

Published on line 06-18-2017

\section{Abstract}

We present an efficient method for the synthesis of polysubstituted imidazoles in the presence of Appel reagent $\left(\mathrm{Ph}_{3} \mathrm{P} / \mathrm{CCl}_{4}\right)$. Tri-substituted imidazoles is synthesized via condensation of aldehydes, benzil and ammonium acetate, and tetra-substituted imidazole is prepared via condensation of aldehydes, benzil, ammonium acetate and primary amines. These protocols allow the simple preparation of the desired products using readily available reagent instead of complex, expensive and toxic reagents under mild reaction conditions in excellent yields.

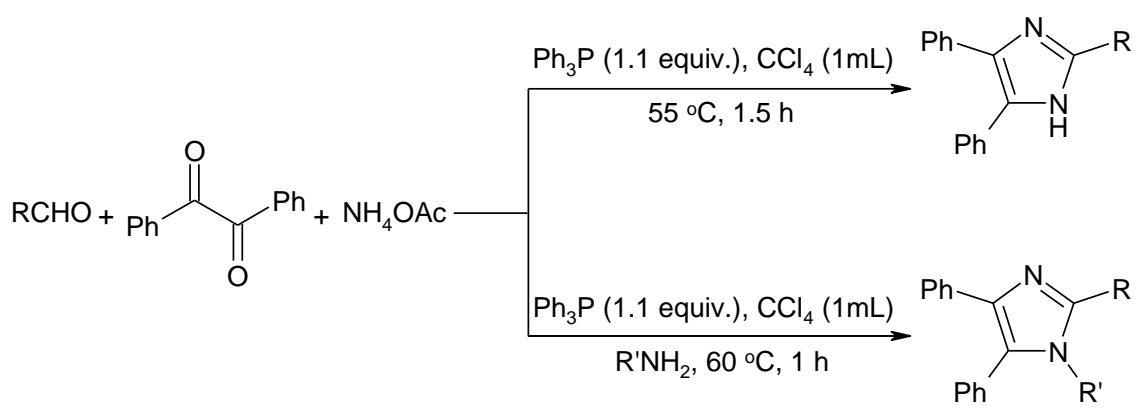

Keywords: Aldehydes, benzil, ammonium acetate, primary amines, Appel reagent, imidazoles 


\section{Introduction}

Imidazole and its derivatives are important class of $N$-heterocycles that occupies a significant place in synthetic and medicinal chemistry. These compounds act as organic catalysts, ${ }^{1}$ precursors of ionic liquids ${ }^{2}$ and carbene ligands, ${ }^{3}$ building blocks of complex meaningful molecules and natural products, ${ }^{4}$ and ligands in metalloenzymes. ${ }^{5}$ The imidazole core exists in many compounds with pharmaceutical and biological activity such as losartan, eprosartan, carnosinemia, histamine, and histidine. ${ }^{6}$ The imidazole-containing compounds have also other useful activities such as anthelmintic, ${ }^{7}$ antifungal, ${ }^{8}$ antiviral activities, ${ }^{9}$ antitubercular, ${ }^{10}$ antitumor, ${ }^{11}$ analgesic, ${ }^{12}$ anti-inflammatory, ${ }^{13}$ and antibacterial activity. ${ }^{14}$
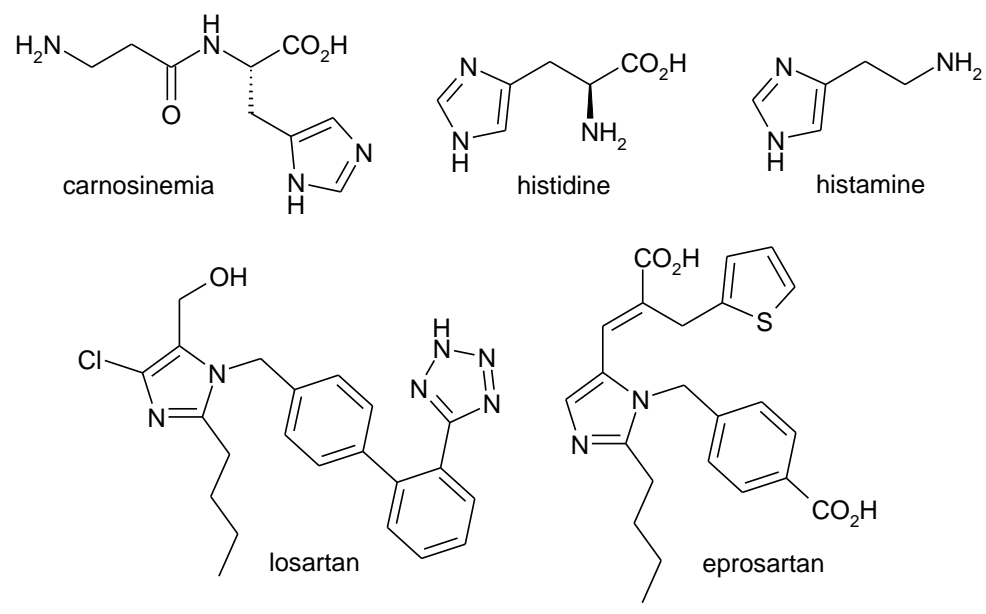

Figure 1. Some examples of pharmaceutical and biological active imidazoles.

Although the broad variety of synthetic routes have been reported to synthesize imidazole derivatives, ${ }^{15,16}$ there are few protocols for preparation of polysubstituted imidazoles. The well-known route for preparation of polysubstituted imidazoles is one-pot reaction between aldehydes, benzil, ammonium acetate and primary amines catalyzed by various catalysts such as $\mathrm{FeCl}_{3} \cdot 6 \mathrm{H}_{2} \mathrm{O},{ }^{17}$ silica gel/ $\mathrm{NaHSO}_{4},{ }^{18} \mathrm{PPA}-\mathrm{SiO}_{2},{ }^{19} \mathrm{BF}_{3} \cdot \mathrm{SiO}_{2}, 20$ silica gel or $\mathrm{HY}$ zeolite, ${ }^{21}$ heteropolyacids, ${ }^{22} \mathrm{HOAc}^{23}{ }^{23} \mathrm{~L}$-proline, ${ }^{24} \mathrm{InCl}_{3} \cdot 3 \mathrm{H}_{2} \mathrm{O},{ }^{25}$ nanocrystalline sulfated zirconia, ${ }^{26} 1,4$ diazabicyclo[2,2,2]octane (DABCO), ${ }^{27} \mathrm{~K}_{5} \mathrm{CoW}_{12} \mathrm{O}_{40} \cdot 3 \mathrm{H}_{2} \mathrm{O},{ }^{28}$ alumina, ${ }^{29}$ ionic liquids, ${ }^{30} \mathrm{HClO}_{4}-\mathrm{SiO}_{2}{ }^{31}$ silicabonded propylpiperazine $\mathrm{N}$-sulfamic acid, 32 and $\mathrm{Zr}(\mathrm{acac})_{4} .{ }^{33}$ However, some of these methods involve the use of toxic and expensive catalysts or media, and have notable disadvantages such as harsh reaction conditions, long reaction times, and moderate yields. Therefore, the development of novel and efficient approaches to generate polysubstituted imidazoles is still desirable.

Due to the chemical and pharmacological significance of imidazoles, we sought to develop a one-pot protocol for the efficient formation of 2,4,5-trisubstituted and 1,2,4,5-tetrasubstituted imidazoles through the addition reaction between aldehydes, benzil, ammonium acetate and primary amines in the presence of $\mathrm{Ph}_{3} \mathrm{P}$ and $\mathrm{CCl}_{4}$ which known as the Appel reagent. Although, the Appel reagent converts an alcohol into the corresponding alkyl halide, we believed that this reagent can be promoted the synthesis of the desired imidazoles. 


\section{Results and Discussion}

To synthesize the 2,4,5-trisubstituted imidazoles, the reaction between 4-methoxybenzaldehyde 1a, benzil 2 and ammonium acetate 3, as a model reaction, was investigated in the presence of various amounts of $\mathrm{Ph}_{3} \mathrm{P}$ and $\mathrm{CCl}_{4}$, at different temperatures. As shown in table 1, the best yield of tri-substituted imidazole 4a was obtained in the presence of 1.1 equiv. of $\mathrm{Ph}_{3} \mathrm{P}$ at $55{ }^{\circ} \mathrm{C}$ in $1 \mathrm{~mL}$ of $\mathrm{CCl}_{4}$ as reactive solvent after $1.5 \mathrm{~h}$ (Table 1 , entry 9, 95\%). In addition, we examined the reaction in various additional solvents such as $\mathrm{CHCl}_{3}, \mathrm{CH}_{2} \mathrm{Cl}_{2}$, toluene, DMF, THF, DMSO, and it was found that although the reaction led to approximately acceptable yields in these solvents, the addition of these solvents did not give the better yield of product (Table 1, entries 1015).

Table 1 Optimization of three-component synthesis of 2,4,5-trisubstituted imidazole $\mathbf{4 a}$ in the presence of Appel reagent ${ }^{a}$

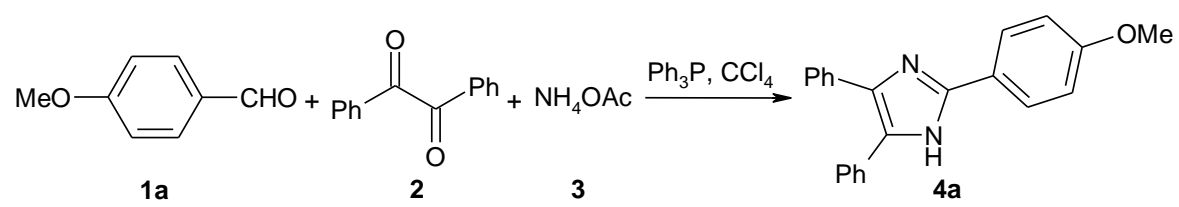

\begin{tabular}{|c|c|c|c|c|c|c|}
\hline Entry & $\mathrm{Ph}_{3} \mathrm{P}$ & $\mathrm{CCl}_{4}$ & Temp. ${ }^{\circ} \mathrm{C}$ & Solvent & Time (h) & $\begin{array}{l}\text { Yield of 4a } \\
(\%)^{b}\end{array}$ \\
\hline 1 & 0.2 equiv. & 0.2 equiv. & r.t & - & 8 & No reaction \\
\hline 2 & 0.2 equiv. & 0.2 equiv. & 40 & - & 8 & 12 \\
\hline 3 & 0.2 equiv. & 0.2 equiv. & 50 & - & 8 & 22 \\
\hline 4 & 0.2 equiv. & 0.2 equiv. & 55 & - & 8 & 25 \\
\hline 5 & 0.5 equiv. & 0.5 equiv. & 55 & - & 8 & 36 \\
\hline 6 & 1.0 equiv. & 1.0 equiv. & 55 & - & 8 & 60 \\
\hline 7 & 1.1 equiv. & 1.1 equiv. & 55 & - & 8 & 65 \\
\hline 8 & 1.1 equiv. & 1.2 equiv. & 55 & - & 4 & 67 \\
\hline 9 & 1.1 equiv. & $1 \mathrm{~mL}$ & 55 & - & 1.5 & 95 \\
\hline 10 & 1.1 equiv. & $1 \mathrm{~mL}$ & 55 & $\mathrm{CH}_{2} \mathrm{Cl}_{2}$ & 1.5 & 70 \\
\hline 11 & 1.1 equiv. & $1 \mathrm{~mL}$ & 55 & $\mathrm{CHCl}_{3}$ & 1.5 & 75 \\
\hline 12 & 1.1 equiv. & $1 \mathrm{~mL}$ & 55 & DMSO & 1.5 & 73 \\
\hline 13 & 1.1 equiv. & $1 \mathrm{~mL}$ & 55 & DMF & 1.5 & 65 \\
\hline 14 & 1.1 equiv. & $1 \mathrm{~mL}$ & 55 & THF & 1.5 & 62 \\
\hline 15 & 1.1 equiv. & $1 \mathrm{~mL}$ & 55 & Toluene & 1.5 & 68 \\
\hline
\end{tabular}

a reaction conditions: use of 4-methoxybenzaldehyde $(1 \mathrm{mmol})$, benzil $(1 \mathrm{mmol})$, ammonium acetate $(2.5$ $\mathrm{mmol}$ ), and appropriate amount of $\mathrm{Ph}_{3} \mathrm{P}$ and $\mathrm{CCl}_{4}$; additional solvent $(3 \mathrm{~mL})$; related temperature and time.

${ }^{\mathrm{b}}$ Isolated yield.

In continuous, we examined the reaction of 4-methoxybenzaldehyde $\mathbf{1 a}$, benzil $\mathbf{2}$, ammonium acetate $\mathbf{3}$, and benzylamine $\mathbf{5 a}$ in the presence of various amount of $\mathrm{Ph}_{3} \mathrm{P}$ and $\mathrm{CCl}_{4}$ under several reaction conditions to generate 1,2,4,5-tetrasubstituted imidazole 6a. As shown in table 2, the best yield of tetra-substituted imidazole 6a was obtained in the presence of 1.1 equiv. of $\mathrm{Ph}_{3} \mathrm{P}$ at $60{ }^{\circ} \mathrm{C}$ in $1 \mathrm{~mL}$ of $\mathrm{CCl}_{4}$ as reactive solvent 
after $1 \mathrm{~h}$ (Table 2, entry 8,92\%). Also, we found that the addition of various solvents such as $\mathrm{CHCl}_{3}, \mathrm{CH}_{2} \mathrm{Cl}_{2}$, toluene, DMF, THF, DMSO in the reaction mixture, did not lead to better yield of product (Table 2, entries 914).

Table 2 Optimization of four-component synthesis of 1,2,4,5-tetrasubstituted imidazole 6a in the presence of Appel reagent ${ }^{a}$

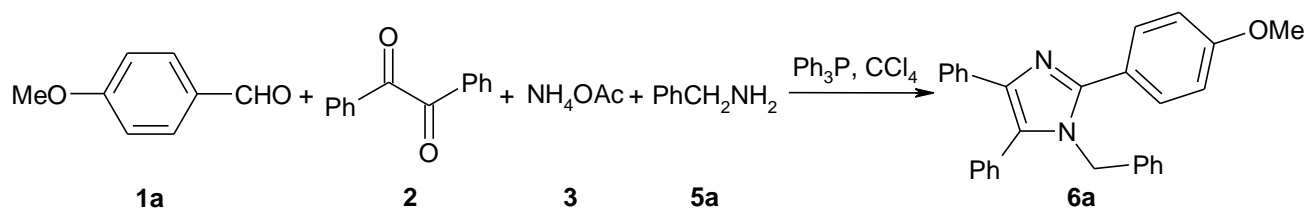

\begin{tabular}{|c|c|c|c|c|c|c|}
\hline Entry & $\mathrm{Ph}_{3} \mathrm{P}$ & $\mathrm{CCl}_{4}$ & Temp. ${ }^{\circ} \mathrm{C}$ & Solvent & Time (h) & $\begin{array}{l}\text { Yield of } 6 a \\
(\%)^{b}\end{array}$ \\
\hline 1 & 0.2 equiv. & 0.2 equiv. & r.t & - & 8 & No reaction \\
\hline 2 & 0.2 equiv. & 0.2 equiv. & 55 & - & 8 & 20 \\
\hline 3 & 0.2 equiv. & 0.2 equiv. & 60 & - & 8 & 26 \\
\hline 4 & 0.5 equiv. & 0.5 equiv. & 60 & - & 8 & 33 \\
\hline 5 & 1.0 equiv. & 1.0 equiv. & 60 & - & 8 & 54 \\
\hline 6 & 1.1 equiv. & 1.1 equiv. & 60 & - & 8 & 62 \\
\hline 7 & 1.1 equiv. & 1.2 equiv. & 60 & - & 6 & 67 \\
\hline 8 & 1.1 equiv. & $1 \mathrm{~mL}$ & 60 & - & 1 & 92 \\
\hline 9 & 1.1 equiv. & $1 \mathrm{~mL}$ & 60 & $\mathrm{CH}_{2} \mathrm{Cl}_{2}$ & 1 & 63 \\
\hline 10 & 1.1 equiv. & $1 \mathrm{~mL}$ & 60 & $\mathrm{CHCl}_{3}$ & 1 & 70 \\
\hline 11 & 1.1 equiv. & $1 \mathrm{~mL}$ & 60 & DMSO & 1 & 67 \\
\hline 12 & 1.1 equiv. & $1 \mathrm{~mL}$ & 60 & DMF & 1 & 58 \\
\hline 13 & 1.1 equiv. & $1 \mathrm{~mL}$ & 60 & THF & 1 & 60 \\
\hline 14 & 1.1 equiv. & $1 \mathrm{~mL}$ & 60 & Toluene & 1 & 65 \\
\hline
\end{tabular}

a reaction conditions: use of 4-methoxybenzaldehyde $(1 \mathrm{mmol})$, benzil $(1 \mathrm{mmol})$, ammonium acetate (1.2 mmol), benzylamine ( $1 \mathrm{mmol})$, and appropriate amount of $\mathrm{Ph}_{3} \mathrm{P}$ and $\mathrm{CCl}_{4}$; additional solvent $(3 \mathrm{~mL})$; related temperature and time.

b Isolated yield.

In order to show the generality and scope of these new protocols, the reactions were performed using various aldehydes 1 and primary amines 5 in the presence of 1.1 equiv. $\mathrm{Ph}_{3} \mathrm{P}$ in $1 \mathrm{~mL} \mathrm{CCl}_{4}$ at convenient temperatures to produce the corresponding polysubstituted imidazoles 4 and 6 (Table 3). 
Table 3 The synthesis of polysubstituted imidazoles $4^{\mathrm{a}}$ and $\mathbf{6}^{\mathrm{b}}$ in the presence of Appel reagent ${ }^{\mathrm{a}}$

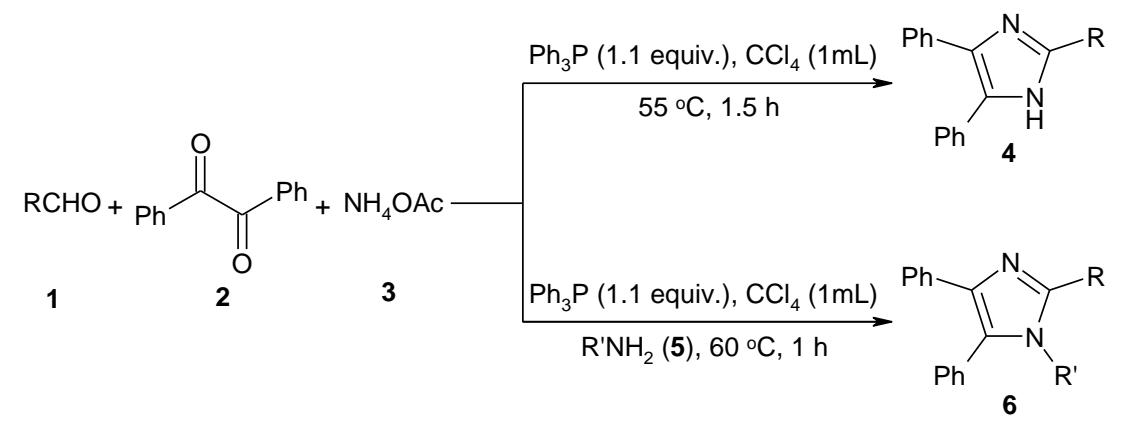

\begin{tabular}{|c|c|c|c|c|c|}
\hline Product & $\mathrm{R}$ & $\mathrm{R}^{\prime}$ & Yield $(\%)^{c}$ & $\mathrm{Mp}\left({ }^{\circ} \mathrm{C}\right)$ & Lit. $\mathrm{mp}\left({ }^{\circ} \mathrm{C}\right)$ \\
\hline $4 a$ & $4-\mathrm{MeOC}_{6} \mathrm{H}_{4}$ & - & 95 & $229-230$ & $228-230^{26}$ \\
\hline $4 b$ & $2-\mathrm{MeOC}_{6} \mathrm{H}_{4}$ & - & 92 & $205-207$ & $204-206^{34}$ \\
\hline 4c & 4- $(\mathrm{Me})_{2} \mathrm{NC}_{6} \mathrm{H}_{4}$ & - & 90 & $257-259$ & $256-259^{24}$ \\
\hline 4d & $4-\mathrm{OHC}_{6} \mathrm{H}_{4}$ & - & 93 & $233-234$ & $234-236^{35}$ \\
\hline $4 e$ & $2-\mathrm{OHC}_{6} \mathrm{H}_{4}$ & - & 91 & $204-206$ & $202-205^{24}$ \\
\hline $4 f$ & $\mathrm{Ph}$ & - & 95 & 275 & $274-276^{26}$ \\
\hline $4 g$ & 4- $\mathrm{MeC}_{6} \mathrm{H}_{4}$ & - & 94 & $233-234$ & $232-234^{26}$ \\
\hline $4 h$ & $4-\mathrm{BrC}_{6} \mathrm{H}_{4}$ & - & 93 & $255-257$ & $254-256^{26}$ \\
\hline $4 i$ & $4-\mathrm{ClC}_{6} \mathrm{H}_{4}$ & - & 94 & $260-261$ & $260-262^{26}$ \\
\hline $4 j$ & $4-\mathrm{NO}_{2} \mathrm{C}_{6} \mathrm{H}_{4}$ & - & 97 & $234-236$ & $234-236^{26}$ \\
\hline $4 k$ & $3-\mathrm{NO}_{2} \mathrm{C}_{6} \mathrm{H}_{4}$ & - & 92 & $308-310$ & $308-309^{36}$ \\
\hline 41 & $2-\mathrm{NO}_{2} \mathrm{C}_{6} \mathrm{H}_{4}$ & - & 94 & 231 & $230-231^{24}$ \\
\hline $6 a$ & $4-\mathrm{MeOC}_{6} \mathrm{H}_{4}$ & $\mathrm{Bn}$ & 92 & $163-165$ & $162-164^{37}$ \\
\hline $6 b$ & 4- $\mathrm{MeOC}_{6} \mathrm{H}_{4}$ & 4- $\mathrm{MeC}_{6} \mathrm{H}_{4}$ & 92 & $177-178$ & $176-178^{38}$ \\
\hline $6 c$ & $4-\mathrm{OHC}_{6} \mathrm{H}_{4}$ & $\mathrm{Bn}$ & 94 & 135 & $134-135^{31}$ \\
\hline $6 d$ & $\mathrm{Ph}$ & $\mathrm{Ph}$ & 96 & $215-216$ & $214-216^{36}$ \\
\hline $6 e$ & $\mathrm{Ph}$ & $\mathrm{Bn}$ & 95 & $164-165$ & $162-164^{39}$ \\
\hline $6 f$ & $4-\mathrm{MeC}_{6} \mathrm{H}_{4}$ & $\mathrm{Ph}$ & 93 & $181-183$ & $182-184^{21}$ \\
\hline $6 g$ & 4- $\mathrm{MeC}_{6} \mathrm{H}_{4}$ & $\mathrm{Bn}$ & 93 & $157-158$ & $156-158^{40}$ \\
\hline $6 h$ & $4-\mathrm{MeC}_{6} \mathrm{H}_{4}$ & 4- $\mathrm{MeC}_{6} \mathrm{H}_{4}$ & 90 & 190-192 & $188-191^{18}$ \\
\hline $6 i$ & $3-\mathrm{NO}_{2} \mathrm{C}_{6} \mathrm{H}_{4}$ & $\mathrm{Bn}$ & 91 & $150-151$ & \\
\hline $6 j$ & $4-\mathrm{BrC}_{6} \mathrm{H}_{4}$ & $\mathrm{Bn}$ & 94 & $172-173$ & $170-172^{31}$ \\
\hline $6 k$ & $4-\mathrm{ClC}_{6} \mathrm{H}_{4}$ & $\mathrm{Bn}$ & 91 & $160-161$ & $160-162^{37}$ \\
\hline 61 & $3-\mathrm{ClC}_{6} \mathrm{H}_{4}$ & $\mathrm{Bn}$ & 91 & $144-145$ & $144-146^{18}$ \\
\hline $6 m$ & $2-\mathrm{ClC}_{6} \mathrm{H}_{4}$ & $\mathrm{Bn}$ & 94 & 141 & $140-141^{31}$ \\
\hline $6 n$ & $4-\mathrm{NO}_{2} \mathrm{C}_{6} \mathrm{H}_{4}$ & 4- $\mathrm{MeC}_{6} \mathrm{H}_{4}$ & 96 & $220-222$ & $219-220^{18}$ \\
\hline 60 & $3-\mathrm{NO}_{2} \mathrm{C}_{6} \mathrm{H}_{4}$ & 4- $\mathrm{MeC}_{6} \mathrm{H}_{4}$ & 90 & $148-150$ & $149-151^{18}$ \\
\hline
\end{tabular}

a Reaction conditions: aldehydes $(1 \mathrm{mmol})$, benzil $(1 \mathrm{mmol})$, ammonium acetate $(2.5 \mathrm{mmol}), \mathrm{Ph}_{3} \mathrm{P}(1.1 \mathrm{mmol})$, $\mathrm{CCl}_{4}(1 \mathrm{~mL}) ; 55^{\circ} \mathrm{C} ; 1.5 \mathrm{~h}$.

${ }^{\mathrm{b}}$ Reaction conditions: aldehydes $(1 \mathrm{mmol})$, benzil $(1 \mathrm{mmol})$, ammonium acetate $(1.2 \mathrm{mmol})$, primary amines (1 $\mathrm{mmol}), \mathrm{Ph}_{3} \mathrm{P}(1.1 \mathrm{mmol}), \mathrm{CCl}_{4}(1 \mathrm{~mL}) ; 60{ }^{\circ} \mathrm{C} ; 1 \mathrm{~h}$.

c Isolated yields. 
All the reactions reached to completion within $1.5 \mathrm{~h}$ for 2,4,5-trisubstituted imidazoles 4 and $1 \mathrm{~h}$ for 1,2,4,5-tetrasubstituted imidazoles $6 .{ }^{1} \mathrm{H}$ NMR analysis of the reaction mixtures clearly indicated formation of the polysubstituted imidazoles 4 and 6 in excellent yields.

The structures of the polysubstituted imidazoles 4 and $\mathbf{6}$ were deduced by melting point determination and from ${ }^{1} \mathrm{H}$ and ${ }^{13} \mathrm{C}$ NMR spectral data.

A proposed mechanism for the formation of the polysubstituted imidazoles $\mathbf{4}$ and $\mathbf{6}$ is depicted in Scheme 1. On the basis of the Appel reaction, ${ }^{41}$ the treatment of triphenylphosphine with carbon tetrachloride leads to form phosphonium ion $\mathbf{7}$, that reacts with aldehydes 1 to form the oxyphosphonium intermediates $\mathbf{8}$. The generation of the oxyphosphonium intermediates $\mathbf{8}$ promotes the nucleophilic addition of ammonium acetate $\mathbf{3}$ via removal of triphenylphosphine oxide and chloride anion to generate the iminium ions $\mathbf{1 0}$. The addition of another ammonium acetate on the iminium ions $\mathbf{1 0}$ gives the intermediates $\mathbf{1 1}$. Then, the condensation of intermediates 11 with benzil $\mathbf{2}$ produces trisubstituted imidazoles 4 by removal of 2 water molecules. In similar pathway, the addition of primary amines 5 onto iminium ions 10 forms intermediates 14 which produces tetrasubstituted imidazol 6 via condensation with benzil.
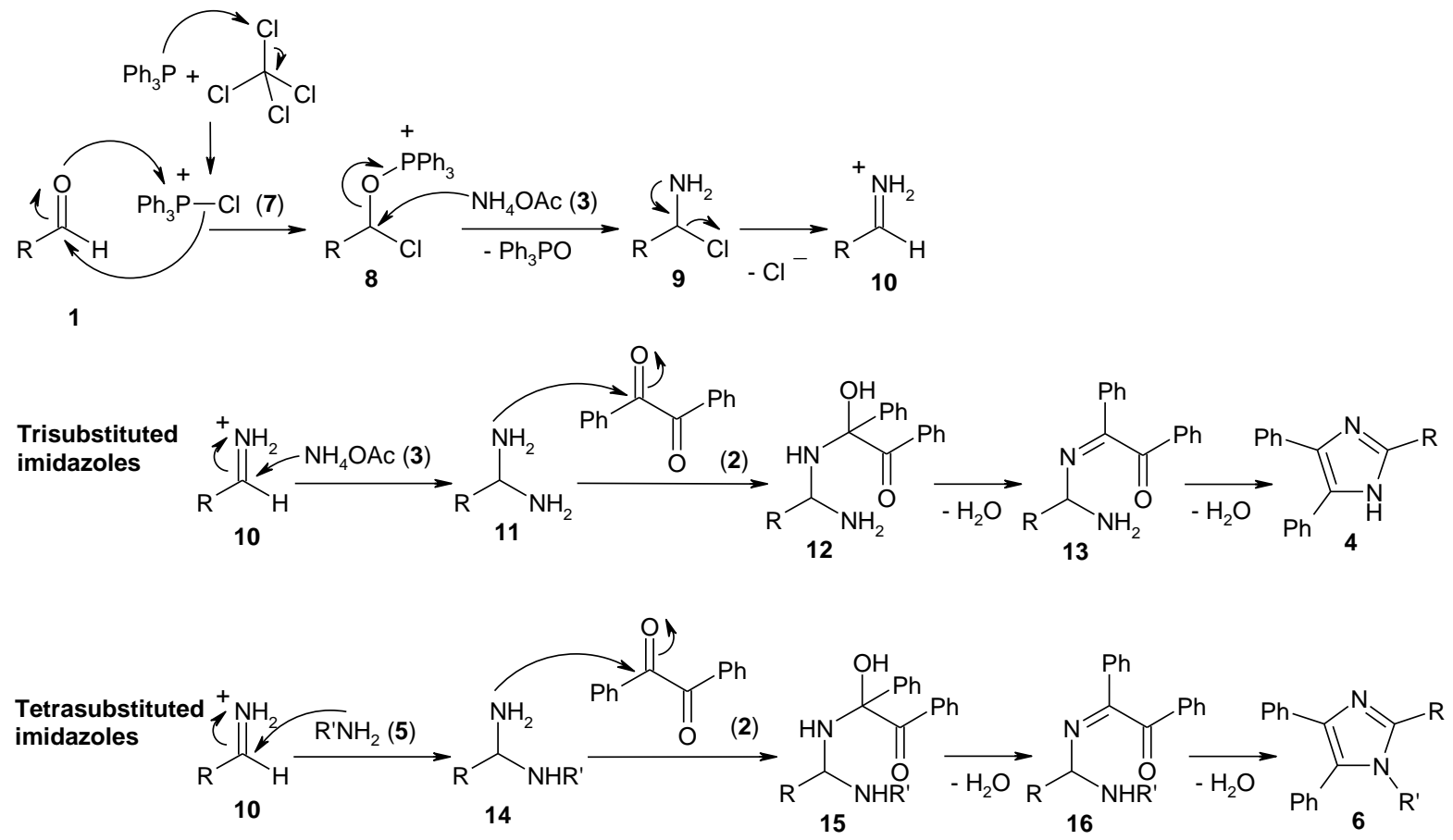

Scheme 1. Proposed mechanism.

\section{Conclusions}

In conclusion, we have developed a one-pot and multicomponent reaction between aldehydes, benzil, ammonium acetate and primary amines in the presence of $\mathrm{Ph}_{3} \mathrm{P}$ and $\mathrm{CCl}_{4}$ which known as Appel reagent. The reactions were carried out under mild reaction conditions and without the use of very high temprature, and complex, toxic and expensive reagents to prepare the polysubstituted imidazoles which are of potential synthetic and pharmacological interest. Use of simple materials, relatively short reaction times, and high yield of the products are the other advantages of our protocol. We believe that the success in this process could 
open the door to the design of diverse reactions and the generation of interesting organic compounds based on treatment of carbonyl groups with Appel reagent.

\section{Experimental Section}

Ammonium acetate, benzil, aldehydes, primary amines, triphenylphosphine and carbon tetrachloride were obtained from Merck (Germany) and Fluka (Switzerland) and were used without further purification. Progress of the reactions was monitored by thin layer chromatography (TLC). Melting points were measured on an Electrothermal 9100 apparatus. ${ }^{1} \mathrm{H}$ and ${ }^{13} \mathrm{C}$ NMR spectra were measured with a Bruker DRX-300 (at 300 and 75 $\mathrm{MHz}$ ) spectrometer using $\mathrm{CDCl}_{3}$ solvent with TMS as an internal standard. Chromatography columns were prepared from Merck silica gel 230-240 meshes.

General procedure for the preparation of 2,4,5-trisubstituted imidazoles 4, exemplified on $4 a$. A mixture of 4-methoxybenzaldehyde $(0.136 \mathrm{~g}, 1 \mathrm{mmol})$, benzil $(0.210 \mathrm{~g}, 1 \mathrm{mmol})$, ammonium acetate $(0.192 \mathrm{~g}, 2.5 \mathrm{mmol})$ and $\mathrm{Ph}_{3} \mathrm{P}(0.280 \mathrm{~g}, 1.1 \mathrm{mmol})$ in $\mathrm{CCl}_{4}(1 \mathrm{~mL})$ was stirred for $1.5 \mathrm{~h}$ at 55 으. After completion of the reaction, the solvent was removed and the residue was purified by column chromatography using $n$-hexane-EtOAc (3:1) as eluent. The solvent was removed to afford the product $\mathbf{4 a}$ as white solid.

The spectral data of some 2,4,5-trisubstituted imidazoles 4 are given next.

2-(4-Methoxyphenyl)-4,5-diphenyl-1H-imidazole (4a) Yield. 0.31 g, 95\%; m.p = 229-230 ㅇ․ ${ }^{1} \mathrm{H} N M R$ (300 $\left.\mathrm{MHz}, \mathrm{CDCl}_{3}\right): \delta=3.80\left(3 \mathrm{H}, \mathrm{s}, \mathrm{OCH}_{3}\right), 7.04(2 \mathrm{H}, \mathrm{d}, J 8.1 \mathrm{~Hz}, 2 \mathrm{CH}), 7.15-7.53(10 \mathrm{H}, \mathrm{m}, 10 \mathrm{CH}), 7.97(2 \mathrm{H}, \mathrm{d}, J 8.1$ $\mathrm{Hz}, 2 \mathrm{CH}), 12.61\left(1 \mathrm{H}\right.$, br s, NH). ${ }^{13} \mathrm{C}$ NMR $\left(75 \mathrm{MHz}, \mathrm{CDCl}_{3}\right): \delta=55.14,114.17,123.55,126.83,127.72,128.38$, $129.01,131.64,135.75,137.19,146.11,159.80$.

2-(2-Hydroxyphenyl)-4,5-diphenyl-1H-imidazole (4e). Yield 0.28 g, 91\%; m.p = 204-206 ㅇ․ ${ }^{1} \mathrm{H} \mathrm{NMR} \mathrm{(300} \mathrm{MHz,}$ $\left.\mathrm{CDCl}_{3}\right): \delta=6.97(1 \mathrm{H}, \mathrm{d}, J 7.6 \mathrm{~Hz}, \mathrm{CH}), 7.14(1 \mathrm{H}, \mathrm{d}, \mathrm{J} 7.6 \mathrm{~Hz}, \mathrm{CH}), 7.29-7.62(11 \mathrm{H}, \mathrm{m}, 11 \mathrm{CH}), 7.65(1 \mathrm{H}, \mathrm{t}, J 7.6 \mathrm{~Hz}$, $\mathrm{CH}), 9.40(1 \mathrm{H}, \mathrm{br} \mathrm{s}, \mathrm{OH}), 12.90(1 \mathrm{H}, \mathrm{br} \mathrm{s}, \mathrm{NH}) .{ }^{13} \mathrm{C} \mathrm{NMR}\left(75 \mathrm{MHz}, \mathrm{CDCl}_{3}\right): \delta=111.42,116.79,118.01,122.08$, $126.43,127.12,127.40,128.11,129.46,144.72,156.37$.

2-(3-Nitrophenyl)-4,5-diphenyl-1H-imidazole (4k). Yield 0.31 g, 92\%; m.p = 308-310 ㅇ. ${ }^{1} \mathrm{H} \mathrm{NMR} \mathrm{(300} \mathrm{MHz,}$ $\left.\mathrm{CDCl}_{3}\right): \delta=7.35-7.58(10 \mathrm{H}, \mathrm{m}, 10 \mathrm{CH}), 7.82(1 \mathrm{H}, \mathrm{t}, J 8.1 \mathrm{~Hz}, \mathrm{CH}), 8.54(1 \mathrm{H}, \mathrm{d}, J 8.1 \mathrm{~Hz}, \mathrm{CH}), 9.02(1 \mathrm{H}, \mathrm{t}, J 1.8 \mathrm{~Hz}$, $\mathrm{CH}), 9.46(1 \mathrm{H}, \mathrm{d}, J 8.1 \mathrm{~Hz}, \mathrm{CH}), 13.13(1 \mathrm{H}, \mathrm{br} \mathrm{s}, \mathrm{NH}) .{ }^{13} \mathrm{C} \mathrm{NMR}\left(75 \mathrm{MHz}, \mathrm{CDCl}_{3}\right): \delta=119.43,122.58,127.05$, $128.41,128.74,130.47,131.23,131.90,143.38,148.32$.

General Procedure for the Preparation of 1,2,4,5-tetrasubstituted imidazoles 6, Exemplified on 6a: A mixture of 4-methoxybenzaldehyde $(0.136 \mathrm{~g}, 1 \mathrm{mmol})$, benzil $(0.210 \mathrm{~g}, 1 \mathrm{mmol})$ ammonium acetate $(0.092 \mathrm{~g}, 1.2$ $\mathrm{mmol})$, benzylamine $(0.107 \mathrm{~g}, 1 \mathrm{mmol})$ and $\mathrm{Ph}_{3} \mathrm{P}(0.280 \mathrm{~g}, 1.1 \mathrm{mmol})$ in $\mathrm{CCl}_{4}(1 \mathrm{~mL})$ was stirred for $1 \mathrm{~h}$ at $60 \stackrel{\circ}{ }$. After completion of the reaction, the solvent was removed and the residue was purified by column chromatography using $n$-hexane-EtOAc $(4: 1)$ as eluent. The solvent was removed to afford the pure product $6 a$ as pale yellow solid.

The spectral data of some 1,2,4,5-tetrasubstituted imidazoles 6 are given next.

1-Benzyl-2-(4-methoxyphenyl)-4,5-diphenyl-1H-imidazole (6a) Yield $0.38 \mathrm{~g}, 92 \% ; \mathrm{mp}=163-165$ o $\mathrm{C} .{ }^{1} \mathrm{H}$ NMR $\left(300 \mathrm{MHz}, \mathrm{CDCl}_{3}\right): \delta=3.80\left(3 \mathrm{H}, \mathrm{s}, \mathrm{OCH}_{3}\right), 5.09\left(2 \mathrm{H}, \mathrm{s}, \mathrm{CH}_{2} \mathrm{Ph}\right), 6.84-7.06(3 \mathrm{H}, \mathrm{m}, 3 \mathrm{CH}), 7.07(2 \mathrm{H}, \mathrm{d}, J 8.0 \mathrm{~Hz}, 2$ $\mathrm{CH}), 7.08-7.56(12 \mathrm{H}, \mathrm{m}, 12 \mathrm{CH}), 7.92(2 \mathrm{H}, \mathrm{d}, J 8.0 \mathrm{~Hz}, \mathrm{CH}) .{ }^{13} \mathrm{C} \mathrm{NMR}\left(75 \mathrm{MHz}, \mathrm{CDCl}_{3}\right): \delta=47.12,54.31,112.89$, $122.38,125.00,125.17,125.65,126.25,127.02,127.52,127.54,128.00,128.67,129.47,130.01,130.16$, $133.53,136.60,136.81,146.92,159.06$. 
1,4,5-Triphenyl-2-p-tolyl-1H-imidazole (6f) Yield $0.36 \mathrm{~g}, 93 \% ; \mathrm{mp}=181-183$ o C. ${ }^{1} \mathrm{H} \mathrm{NMR}(300 \mathrm{MHz}, \mathrm{CDCl} 3): \delta=$ $2.30\left(3 \mathrm{H}, \mathrm{s}, \mathrm{CH}_{3}\right), 7.11(2 \mathrm{H}, \mathrm{d}, J 7.9 \mathrm{~Hz}, 2 \mathrm{CH}), 7.19(1 \mathrm{H}, \mathrm{t}, J 7.5 \mathrm{~Hz}, \mathrm{CH}), 7.24-7.31(12 \mathrm{H}, \mathrm{m}, 12 \mathrm{CH}), 7.35(2 \mathrm{H}, \mathrm{d}, J$ $7.5 \mathrm{~Hz}, 2 \mathrm{CH}), 7.54(2 \mathrm{H}, \mathrm{d}, J 7.9 \mathrm{~Hz}, 2 \mathrm{CH}) .{ }^{13} \mathrm{C} \mathrm{NMR}\left(75 \mathrm{MHz}, \mathrm{CDCl}_{3}\right): \delta=21.56,127.18,128.45,129.03,129.06$, 129.24, 129.52, 129.60, 129.66, 123.24, 131.35, 132.02, 132.17, 135.36, 137.65, 137.76, 138.58, 147.08.

1-Benzyl-2-(3-nitrophenyl)-4,5-diphenyl-1H-imidazole (6i) Yield 0.39 g, 91\%; mp = 150-151 ㅇ․ ${ }^{1} \mathrm{H} \mathrm{NMR}(300$ $\left.\mathrm{MHz}, \mathrm{CDCl}_{3}\right): \delta=5.24\left(2 \mathrm{H}, \mathrm{s}, \mathrm{CH}_{2} \mathrm{Ph}\right), 6.84-7.12(5 \mathrm{H}, \mathrm{m}, 5 \mathrm{CH}), 7.20(2 \mathrm{H}, \mathrm{d}, J 7.5 \mathrm{~Hz}, 2 \mathrm{CH}), 7.23-7.54(6 \mathrm{H}, \mathrm{m}, 6$ $\mathrm{CH}), 7.59(1 \mathrm{H}, \mathrm{t}, J 7.9 \mathrm{~Hz}, \mathrm{CH}), 7.70(2 \mathrm{H}, \mathrm{t}, J 7.5 \mathrm{~Hz}, 2 \mathrm{CH}), 8.10(1 \mathrm{H}, \mathrm{d}, J 7.9 \mathrm{~Hz}, \mathrm{CH}), 8.29(1 \mathrm{H}, \mathrm{d}, J 7.9 \mathrm{~Hz}, \mathrm{CH})$, $8.59(1 \mathrm{H}, \mathrm{s}, \mathrm{CH}) .{ }^{13} \mathrm{C} \mathrm{NMR}\left(75 \mathrm{MHz}, \mathrm{CDCl}_{3}\right): \delta=47.38,122.35,122.62,124.70,125.70,125.65,126.73,127.21$, $127.87,128.06,128.58,129.43,129.98,130.27,131.63,133.05,133.50,135.86,144.33,147.17$.

1-(4-Methylphenyl)-2-(3-nitrophenyl)-4,5-diphenyl-1H-imidazole (6o) Yield $0.39 \mathrm{~g}, 90 \% ; \mathrm{mp}=148-150$ 으. ${ }^{1} \mathrm{H}$ NMR (300 MHz, CDCl $): \delta=2.31\left(3 \mathrm{H}, \mathrm{s}, \mathrm{CH}_{3}\right), 6.87-7.39(14 \mathrm{H}, \mathrm{m}, 14 \mathrm{CH}), 7.54(1 \mathrm{H}, \mathrm{t}, J 8.0 \mathrm{~Hz}, \mathrm{CH}), 8.16(1 \mathrm{H}, \mathrm{d}, J$ $8.0 \mathrm{~Hz}, \mathrm{CH}), 8.30(1 \mathrm{H}, \mathrm{d}, J 8.0 \mathrm{~Hz}, \mathrm{CH}), 8.55(1 \mathrm{H}, \mathrm{s}, \mathrm{CH}) .{ }^{13} \mathrm{C} \mathrm{NMR}\left(75 \mathrm{MHz}, \mathrm{CDCl}_{3}\right): \delta=21.17,122.67,123.50$, $127.00,127.31,127.96,128.22,128.85,129.05,131.01,131.90,132.27,133.64,134.37,138.68,139.12$, 144.36, 147.98 .

\section{Acknowledgements}

This study was supported by the Research Council of Tehran University of Medicinal Sciences (TUMS) and Iran National Science Foundation (INSF).

\section{References}

1. Li, Y.; Giulionatti, M.; Houghten, R. A. Org. Lett. 2010, 12, 2250-2253, and references therein. https://doi.org/10.1021/ol100596p

2. Dupont, J.; de Souza, R. F.; Suarez, P. A. Chem. Rev. 2002, 102, 3667-3692. https://doi.org/10.1021/cr010338r

3. Hahn, F. E.; Jahnke, M. C. Angew. Chem. 2008, 120, 3166-3216. https://doi.org/10.1002/ange.200703883

4. Ueda, S.; Su, M.; Buchwald, S. L. J. Am. Chem. Soc. 2012, 134, 700-706, and references therein. https://doi.org/10.1021/ja2102373

5. Holm, R. H.; Kennepohl, P.; Solomon, E. I. Chem. Rev. 1996, 96, 2239-2314. https://doi.org/10.1021/cr9500390

6. Boersma, C.; Atthobari, J.; Gansevoort, R. T.; de Jong, P. E.; de Zeeuw, D.; Annemans, L. J.; Postma, M. J. Pharmacoeconomics 2006, 24, 523-535.

https://doi.org/10.2165/00019053-200624060-00001

7. Dutta, S. Acta Pharm. 2010, 60, 229-235. https://doi.org/10.2478/v10007-010-0011-1

8. Dahiya, R. Sci. Pharm. 2008, 76, 217-239. https://doi.org/10.3797/scipharm.0803-04

9. Cheng, J.; Xie, J.; Luo, X. Bioorg. Med. Chem. Lett. 2005, 15, 267-269. https://doi.org/10.1016/j.bmcl.2004.10.087 
10. Narayanan, S.; Vangapandu, S.; Jain, R. Bioorg. Med. Chem. Lett. 2001, 11, 1133-1136. https://doi.org/10.1016/S0960-894X(01)00154-8

11. Wang, L.; Woods, K. W.; Li, Q.; Barr, K. J.; McCroskey, R. W.; Hannick, S. M.; Gherke, L.; Credo, R. B.; Hui, Y. H.; Marsh, K.; Warner, R. J. Med. Chem. 2002, 45, 1697-1711.

https://doi.org/10.1021/jm010523x

12. Sondhi, S. M.; Jain, S.; Dinodia, M.; Kumar, A. Med. Chem. 2008, 4, 146-154, and references therein. https://doi.org/10.2174/157340608783789194

13. Li, Q.; Hu, Q.; Wang, X.; Zong, Y.; Zhao, L.; Xing, J.; Zhou, J.; Zhang, H. Chem. Biol. Drug Des. 2015, 86, 509-516.

https://doi.org/10.1111/cbdd.12513

14. Matysiak, J.; Niewiadomy, A.; Krajewska-Kułak, E.; Mącik-Niewiadomy, G. II Farmaco 2003, 58, $455-461$. https://doi.org/10.1016/S0014-827X(03)00046-6

15. Gribble, G. W.; Joule, J. A.; Gilchrist, T. L. Progress in heterocyclic chemistry; Elsevier: Oxford, 2001-2005, and references therein.

16. Zhu, Y.; Li, C.; Zhang, J.; She, M.; Sun, W.; Wan, K.; Wang, Y.; Yin, B.; Liu, P.; Li, J. Org. Lett. 2015, 17, 3872-3875, and references therein.

https://doi.org/10.1021/acs.orglett.5b01854

17. Heravi, M. M.; Derikvand, F.; Haghighi, M. Monatsh. Chem. 2008, 139, 31-33.

https://doi.org/10.1007/s00706-007-0736-9

18. Karimi, A. R.; Alimohammadi, Z.; Azizian, J.; Mohammadi, A. A.; Mohammadizadeh, M. R. Catal. Commun. 2006, 7, 728-732.

https://doi.org/10.1016/i.catcom.2006.04.004

19. Montazeri, N.; Pourshamsian, K.; Khoddadi, M.; Khoddadi, K. Orient. J. Chem. 2011, 27, 1023-1027.

20. Sadeghi, B.; Mirjalili, B. B. F.; Hashemi, M. M. Tetrahedron Lett. 2008, 49, 2575-2577.

https://doi.org/10.1016/j.tetlet.2008.02.100

21. Balalaie, S.; Arabanian, A. Green Chem. 2000, 2, 274-276.

https://doi.org/10.1039/b0062010

22. Heravi, M. M.; Derikvand, F.; Bamoharram, F. F. J. Mol. Catal. A Chem. 2007, 263, 112-114.

https://doi.org/10.1016/i.molcata.2006.08.048

23. Schubert, H.; Stodolka, H. J. Prakt. Chem. 1963, 22, 130-139.

https://doi.org/10.1002/prac.19630220303

24. Samai, S.; Nandi, G. C.; Singh, P.; Singh, M. S. Tetrahedron 2009, 65, 10155-10161.

https://doi.org/10.1016/i.tet.2009.10.019

25. Kidwai, M.; Mothsra, P.; Bansal, V.; Somvanshi, R. K.; Ethayathulla, A. S.; Dey, S.; Singh, T. P. J. Mol. Catal. A Chem. 2007, 265, 177-182.

https://doi.org/10.1016/i.molcata.2006.10.009

26. Teimouri, A.; Chermahini, A. N. J. Mol. Catal. A Chem. 2011, 346, 39-45.

https://doi.org/10.1016/i.molcata.2011.06.007

27. Murthy, S. N.; Madhav, B.; Nageswar, Y. V. D. Tetrahedron Lett. 2010, 51, 5252-5257. https://doi.org/10.1016/i.tetlet.2010.07.128

28. Nagarapu, L.; Apuri, S.; Kantevari, S. J. Mol. Catal. A Chem. 2007, 266, 104-108. https://doi.org/10.1016/j.molcata.2006.10.056

29. Usyatinsky, A. Y.; Khmelnitsky, Y. L. Tetrahedron Lett. 2000, 41, 5031-5034.

https://doi.org/10.1016/S0040-4039(00)00771-1 
30. Zang, H.; Su, Q.; Mo, Y.; Cheng, B. W.; Jun, S. Ultrason. Sonochem. 2010, 17, 749-751. https://doi.org/10.1016/j.ultsonch.2010.01.015

31. Kantevari, S.; Vuppalapati, S. V.; Biradar, D. O.; Nagarapu, L. J. Mol. Catal. A Chem. 2007, 266, $109-113$. https://doi.org/10.1016/i.molcata.2006.10.048

32. Niknam, K.; Deris, A.; Naeimi, F.; Majleci, F. Tetrahedron Lett. 2011, 52, 4642-4645. https://doi.org/10.1016/i.tetlet.2011.06.105

33. Hasaninejad, A.; Zare, A.; Shekouhy, M.; Ameri Rad, J. J. Comb. Chem. 2010, 12, 844-849. https://doi.org/10.1021/cc100097m

34. Karami, B.; Dehghani, F. M.; Eskandari, K. Croat. Chem. Acta, 2012, 85, 147-153. https://doi.org/10.5562/cca1979

35. Banothu, J.; Gali, R.; Velpula, R.; Bavantula, R. Arab. J. Chem., 2013. http://dx.doi.org/10.1016/i.arabjc.2013.10.022.

36. Wang, L. M.; Wang, Y. H.; Tian, H.; Yao, Y. F.; Shao, J. H.; Liu, B. J. Fluorine Chem. 2006, 127, $1570-1573$. https://doi.org/10.1016/j.jfluchem.2006.08.005

37. Mohammadi, A.; Keshvari, H.; Sandaroos, R.; Maleki, B.; Rouhi, H.; Moradi, H.; Sepehr, Z.; Damavandi, S. Appl. Catal. A. 2012, 429, 73-78. https://doi.org/10.1016/i.apcata.2012.04.011

38. Ray, S.; Das, P.; Bhaumik, A.; Dutta, A.; Mukhopadhyay, C. Appl. Catal., A. 2013, 458, $183-195$. https://doi.org/10.1016/i.apcata.2013.03.024

39. Reddy, P. P.; Mukkanti, K.; Purandhar, K. Rasayan J. Chem. 2010, 3, 335-340.

40. Karimi, A. R.; Alimohammadi, Z.; Amini, M. M. Mol. Div. 2010, 14, 635-641. https://doi.org/10.1007/s11030-009-9197-x

41. Appel, R. Angew. Chem. Int. Ed. 1975, 14, 801-811. https://doi.org/10.1002/anie.197508011 